\title{
PPROM at 15 Weeks of Gestation: A Story of Hope
}

\author{
Aziz Rodolphe ${ }^{1,2}$, Halimeh Rawad ${ }^{1,2, ~ *, ~ F e g h a l i ~ J o e ~}{ }^{1,2}$ \\ ${ }^{1}$ Department of Obstetrics and Gynecology, Saint George Hospital University Medical Center, Beirut, Lebanon \\ ${ }^{2}$ Faculty of Medicine, University of Balamand, Beirut, Lebanon
}

Email address:

rawad.halimeh@gmail.com (H. Rawad)

${ }^{*}$ Corresponding author

\section{To cite this article:}

Aziz Rodolphe, Halimeh Rawad, Feghali Joe. PPROM at 15 Weeks of Gestation: A Story of Hope. Journal of Gynecology and Obstetrics. Vol. 9, No. 5, 2021, pp. 162-166. doi: 10.11648/j.jgo.20210905.15

Received: August 18, 2021; Accepted: September 7, 2021; Published: September 30, 2021

\begin{abstract}
Spontaneous preterm premature rupture of membranes that occurs before 20 weeks of gestation is a rare complication that is usually followed by miscarriage. There are various risk factors, including infection, inflammation, trauma, and it can sometimes be idiopathic. If PPROM happens as early as 15 weeks of gestation, termination of the pregnancy is usually the method of choice. Several risks affect the fetus and the mother if the mother decides to continue her pregnancy. The fetal musculoskeletal, gastrointestinal, respiratory, and nervous systems are mainly affected, and fetal death occurs in most cases. In patients who decide to take the risk and continue their pregnancy, conservative management with antibiotics and monitoring is usually required. The end result is either chorioamnionitis or spontaneous expulsion. This case presents a rare outcome of pregnancy after spontaneous preterm premature rupture of membranes at 15 weeks of gestation and delivery of a healthy living baby at term. The management of this case throughout pregnancy will be presented along with a literature review.
\end{abstract}

Keywords: PPROM, Early Gestation, Conservative Management, Live Baby

\section{Introduction}

Preterm premature rupture of membranes (PPROM) is the rupture of the amniotic membranes that occurs before 37 weeks of gestation. It also happens prior to the onset of labor. It can occur prior to 26 weeks of gestation and contributes to a higher risk of prematurity, leading to maternal and fetal complications [1]. Patients generally present with spontaneous leakage of fluids, with no other associated symptoms.

The diagnosis of PPROM can be done by performing a speculum exam, where pooling can be detected with a positive Fern test or positive nitrazine test. A false-positive result can occur especially when contaminants are present such as semen, blood, and vaginal antiseptics [2]. Recently, placental alpha macroglobulin-1 test (AFP) and insulin-like growth factor binding protein 1 (IGFBP1) are used to diagnose ruptured membranes [2]. Leakage of urine and heavy vaginal secretions are commonly diagnosed especially in advanced gestational age, and patients are therefore reassured and discharged.

The incidence of PPROM in all pregnancies is about 2-
$3 \%$ and comprises $30-40 \%$ of preterm deliveries [3]. PPROM at 16 through 26 weeks of gestation complicates approximately $1 \%$ of pregnancies in the United States and is associated with significant risk of neonatal morbidity and mortality [4]. Perinatal mortality is high if PPROM occurs when fetuses are of previable gestational age. Moretti and Sibai reported an overall survival rate of $50 \%$ to $70 \%$ after delivery at 24 to 26 weeks of gestation [5]. Another study done on patients with PPROM between 14 to 24 weeks of gestation concluded that women with PPROM before 20 weeks of gestation, have a poor prognosis with extremely low fetal survival. In addition, no survival advantage was seen if the patient had a longer duration of pregnancy [6].

This case shows that exceptionally, the survival of a fetus along with a healthy maternal and fetal outcome can be possible for patients presenting with PPROM at less than 20 weeks of gestation. 


\section{Case Presentation}

This is the case of a 31-year-old pregnant patient, G3P0111 with a previous history of a cesarean section at 35 weeks of gestation for spontaneous PPROM and a previous open myomectomy, presenting at 15 weeks and 4 days with PPROM. Her medical history consists of hypothyroidism status post thyroidectomy that is well controlled. Review of systems was unremarkable. The patient denies smoking and alcohol consumption. Her medications include prenatal vitamins and levothyroxine $125 \mathrm{mg}$ daily. Vital signs were within normal limits. Pooling and leakage of clear fluids from the cervical os was seen on speculum exam.

Blood tests were done on admission showing $\mathrm{Hb} 11.8 \mathrm{~g} / \mathrm{dl}$, WBC $1010^{9} / \mathrm{L}$, PLT $26110^{9} / \mathrm{L}$, TSH $1.0 \mathrm{microU} / \mathrm{ml}$, CRP negative, urine analysis and culture were negative, and the vaginal culture was negative.

The obstetrical ultrasound done at presentation showed a singleton gestation with a crown rump length (CRL) measuring $98 \mathrm{~mm}$, positive fetal heart rate but with complete anhydramnios (Figure 1). The nuchal thickness was normal, and the cervix was closed measuring $4 \mathrm{~cm}$.

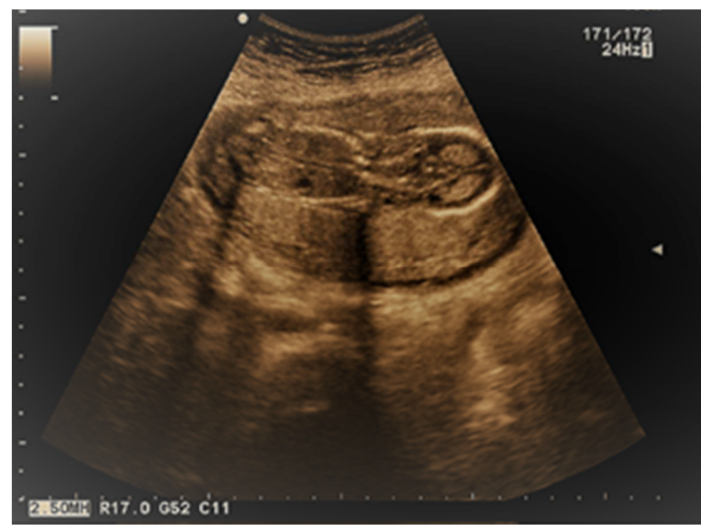

Figure 1. Complete Anhydramnios.

Ampicillin $500 \mathrm{mg}$ tid for 1 week was started along with dydrogesterone bid. The patient was admitted for constant inhospital monitoring. Serial CBC, CRP and cultures were done and were negative. She was discharged on day 7 on an out-patient follow up that consisted of weekly labs and ultrasound. The patient was also instructed to report any symptom of fever, chills, foul smelling vaginal discharge or vaginal bleeding related to her condition to her treating physician.

The patient was followed up closely from 15 to 21 weeks of gestation when a morphological scan was done showing a fetus with normal morphology except for bilateral talipes (Figure 2). The fetus had normal kidney morphology (Figure 3) with normal ultrasonographic bladder parameters (Figure 5), there was no ultrasonographic evidence of neural tube defect (Figure 4) and umbilical artery doppler was normal (two umbilical arteries seen) with no ultrasonographic evidence of placental insufficiency (Figure 5). Normal amount of amniotic fluid was seen, and the fetus had normal growth.
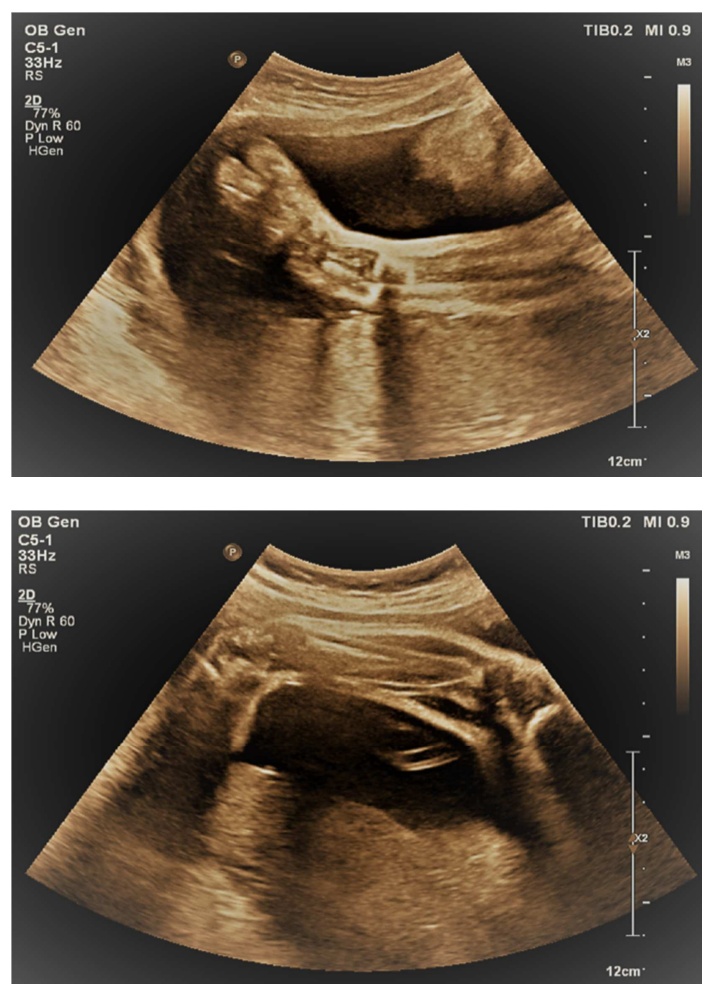

Figure 2. Bilateral Talipes with normal amniotic fluid at 21 weeks of gestational age.

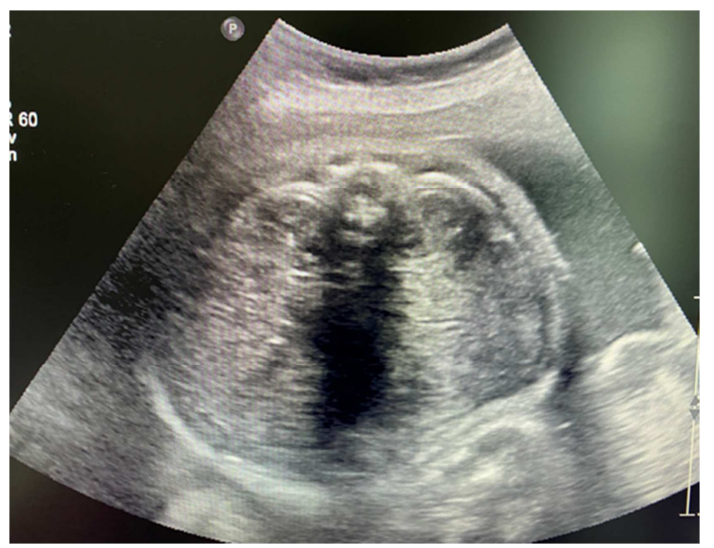

Figure 3. Bilateral Kidneys with no ultrasonographic evidence of Hydronephrosis.

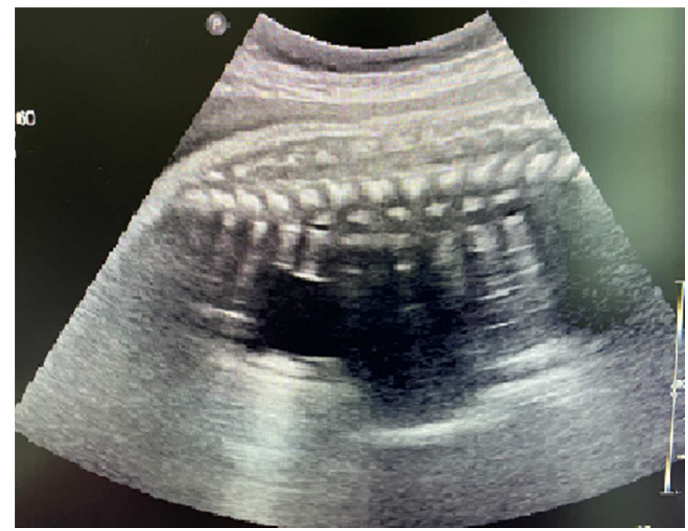

Figure 4. Normal Vertebrae with no ultrasonographic evidence of Neural Tube Defects. 


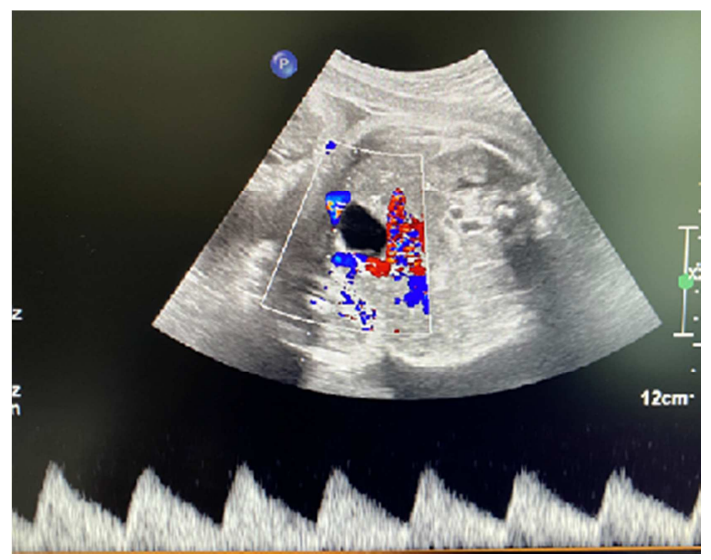

Figure 5. Normal Bladder and normal Umbilical artery Doppler.
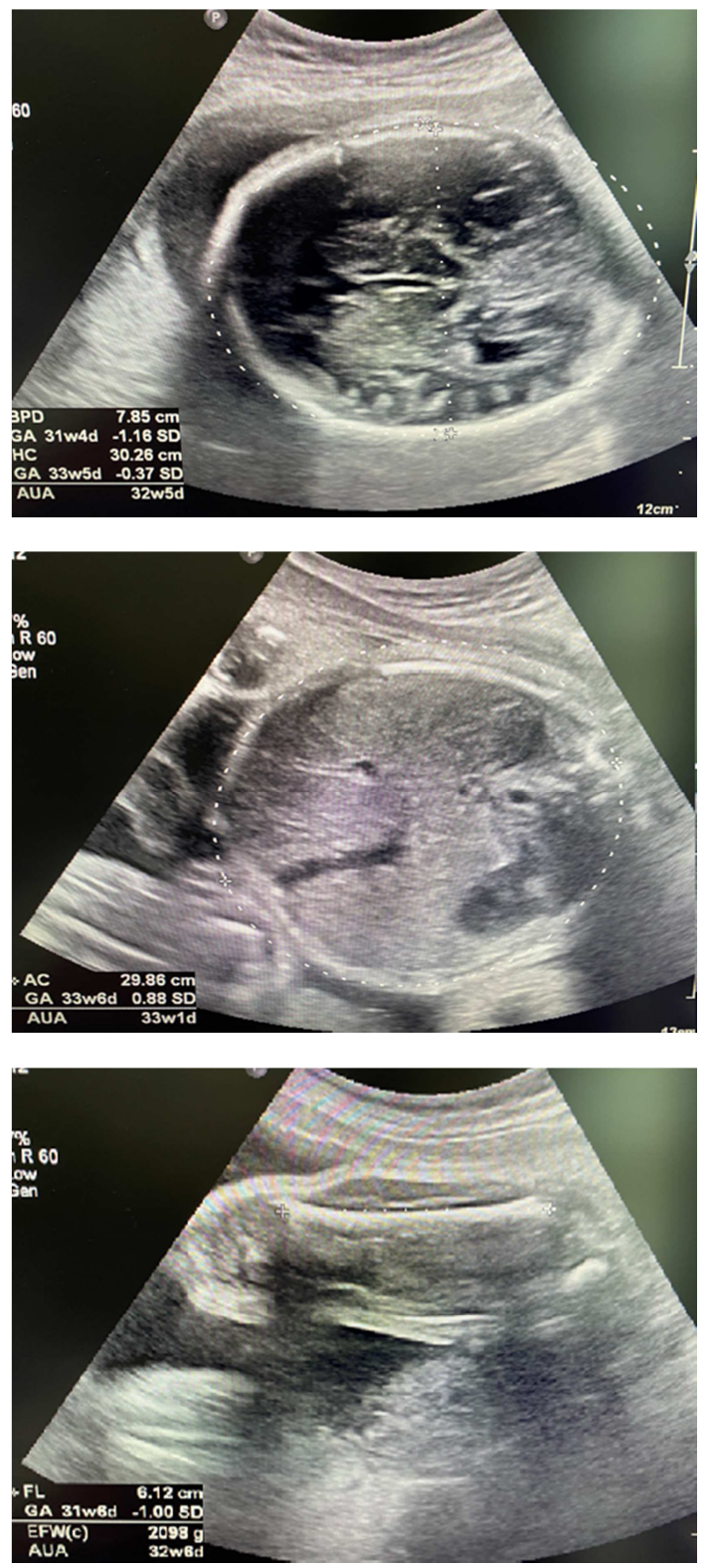

Figure 6. Fetal Biometry with an estimated fetal weight of $2098 \mathrm{~g}$ corresponding to $32 w 6 d$.

The patient reported constant leakage of clear fluids until
30 weeks of gestation after which it stopped (No foulsmelling discharge or bleeding reported). The same management was continued until the leakage stopped, then a routine follow up was continued and the patient was given a full course of dexamethasone for fetal lung maturity at 28 weeks of gestation. She underwent $3^{\text {rd }}$ trimester growth scan at 33 weeks that showed appropriate growth and an EFW of $2098 \mathrm{~g}$ (Figure 6) with normal umbilical artery doppler and normal amniotic fluid index (AFI).

The group B Streptococcus Culture was done at 36 weeks, and it was negative. The patient presented at 37 weeks in labor and delivered a healthy baby boy by Cesarean section. The baby had congenital talipes equinovarus but was eventually discharged home with the mother on day 3 post operation.

A follow up was done 3 months after delivery assuring that the child is healthy and did not undergo any orthopedic surgery. Alignment was done using a foot cast coupled with physical therapy sessions. (Figure 7)
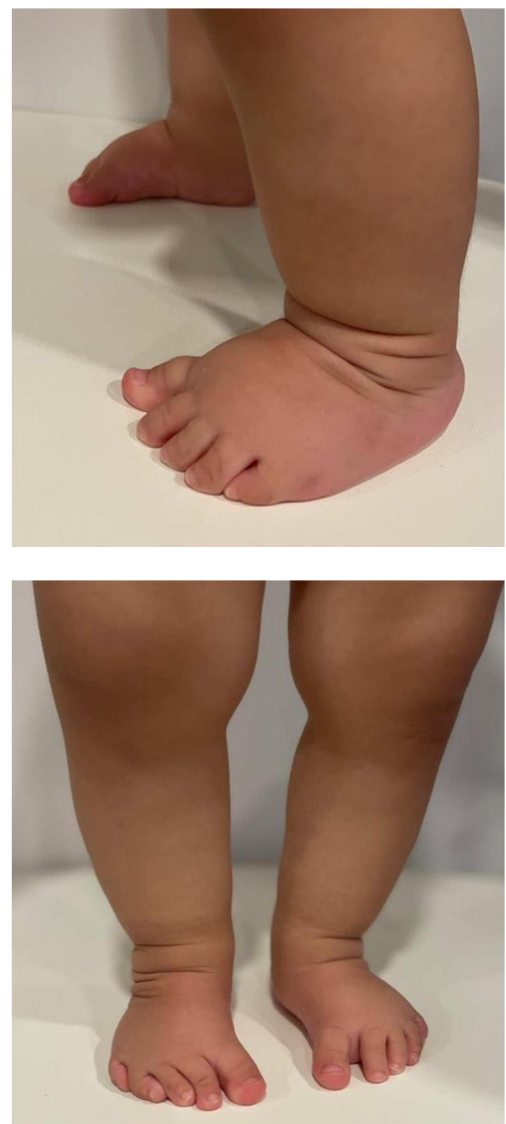

Figure 7. Corrected Bilateral Club Foot 3 months post-delivery.

\section{Discussion}

It is highly significant if PPROM occurs prior to 26 weeks of gestation. It can lead to many fetal, and neonatal complications including respiratory distress syndrome, neonatal sepsis, and fetal death. Surviving neonates may also have bronchopulmonary dysplasia, necrotizing enterocolitis, and contractures [7]. Maternal complications also can be 
severe including chorioamnionitis and postpartum endometritis.

Numerous risk factors are associated with PPROM. Infection (mostly bacterial infection) is the major risk factor associated with PPROM. On one hand, the prostaglandins act by stimulating the contraction of the uterus and on the other hand, the metalloproteases relax and soften the cervix, leading to membrane rupture [8]. Other risk factors include race/ ethnicity, parity, history of preterm birth, in utero exposure to diethylstilbesterol, pregnancy complications such as gestational and pre-existing diabetes mellitus, antepartum bleeding and anemia, maternal weight gain, smoking, illegal drug use, and uterine distention [1]. The patient presented above had a history of PPROM at 35 weeks which increases her risk of PPROM. The Odds Ratio for recurrent spontaneous preterm birth with ruptured membranes is 5.5 (95\% CI 3.2, 9.4) [9].

In 1988, Moretti and Sibai evaluated maternal and neonatal outcomes for 118 mid trimester cases of PROM that were managed expectantly and occurred between 16 and 26 weeks of gestation. The mean gestational age at the onset of PROM was 23.1 weeks with a mean latency period of 13 days. Sixty-seven percent of the patients delivered within 1 week following PROM. Perinatal mortality was recorded at $67.7 \%[5]$.

It is of utmost importance to rule out all other causes of oligohydramnios/anhydramnios prior to establishing the diagnosis of PPROM, as management differs significantly. Some of the causes of oligohydramnios include renal abnormalities affecting fetal urine output such as renal agenesis, renal dysplasia, posterior urethral valve, polycystic kidneys, multicystic dysplastic kidneys and urethral atresia. The patient presented above had no ultrasonographic evidence of any renal abnormalities (Figures 5, 7). Other causes of oligohydramnios include intrauterine growth restriction (IUGR). The patient presented above had a fetus with normal growth and fetal biometry appropriate to gestational age (Figure 3) and no evidence of placental insufficiency or IUGR (Figure 7). Other causes include chromosomal abnormalities mainly trisomy 18, trisomy 13 and triploidy. The patient presented above had a low risk for trisomies based on maternal and fetal characteristics. Potter sequence was also ruled out through several ultrasound examinations and the patient had not had any prostaglandin inhibitors such as indomethacin. To note, the patient had no ultrasonographic signs of neural tube defects (Figures 4, 6).

Pregnant women presenting with PPROM are managed according to gestational age at the time of presentation, maternal and fetal statuses. Conservative approach is preferred when a woman presents before 34 weeks with PPROM but has no maternal or fetal complications. Administration of antibiotics is mandatory for pregnancy prolongation and for the prevention of chorioamnionitis, and corticosteroids are essential to ensure fetal lung maturation. There are few published data looking into the management and outcomes of gestations complicated with PPROM at extreme gestational age [1]. It is evident that daily non stress test and daily biophysical profile, have no clinical significance at predicting the infectious complications after PPROM [10]. Maternal serum C Reactive Protein (CRP) has been shown to be the most accurate predictor of chorioamnionitis in PPROM patients [11]. Hence, chorioamnionitis is a diagnosis that depends on several clinical [12] and histological [13] predictor factors. Perinatal survival is largely dependent on fetuses' gestational age at PPROM. When fetuses are of viable gestational age, the immediate perinatal survival rate we observed was $100 \%$, but outcomes are discouraging when fetal membranes rupture before 23 weeks of gestation.

Decisions concerning early induction of labor or cesarean delivery are also complex [14]. Clinical decisions to prolong latency period is also very critical particularly for pregnancies with PPROM at the limit of viability $[15,16]$.

\section{Conclusion}

Over the past 20 years, obstetrics and neonatal care have been working on improving perinatal outcomes in patients presenting with ruptured membranes before 23 weeks of gestation, however the progress remains minimal, and outcomes are still poor. If the patient aims for expectant management, the physician should counsel the pregnant woman about the poor neonatal outcomes and the maternal and fetal risks of expectant management. The physician should also discuss all the anticipated risks of delivering at an early gestational age. Alternatively, maternal morbidity does not seem to increase when the patient agrees on aggressive expectant management. Most of these women will deliver at a previable gestational age, either by going into spontaneous labor, or will need to be delivered due to maternal risks. Furthermore, an extremely poor prognosis for the fetus/neonate is often presented to the patient and termination of pregnancy is frequently recommended. It is of utmost importance to counsel women who present with PPROM at previable gestational age, but challenges remain in the approach that should be used. This case shows that extreme PPROM could end with beneficial outcomes. However, constant monitoring and serial blood tests and obstetrical ultrasounds are recommended routinely with close outpatient followup.

\section{References}

[1] Al Riyami, N., Al-Ruheili, I., Al-Shezawi, F. and Al-Khabori, M., 2013. Extreme Preterm Premature Rupture of Membranes: Risk Factors and Feto Maternal Outcomes. Oman Medical Journal, 28 (2), pp. 108-111.

[2] Tchirikov, M., Schlabritz-Loutsevitch, N., Maher, J., Buchmann, J., Naberezhnev, Y., Winarno, A., \& Seliger, G. (2017). Mid-trimester preterm premature rupture of membranes (PPROM): etiology, diagnosis, classification, international recommendations of treatment options and outcome. Journal of Perinatal Medicine, 46 (5), 465-488. doi: 10.1515/jpm-2017-0027. 
[3] Mercer, B., 2003. Preterm Premature Rupture of the Membranes. Obstetrics \& Gynecology, 101 (1), pp. 178-193.

[4] Yang, L., Hendricks, S., Taylor, D., Hume, R., Calhoun, B. and Kaufman, H., 2003. Active Expectant Management of Second-Trimester Preterm Premature Rupture of Membranes. Obstetrics \& Gynecology, 101 (Supplement), p. 36S.

[5] Moretti, M. and Sibai, B., 1988. Maternal and perinatal outcome of expectant management of premature rupture of membranes in the midtrimester. American Journal of Obstetrics and Gynecology, 159 (2), pp. 390-396.

[6] Falk, S., Campbell, L., Lee-Parritz, A., Cohen, A., Ecker, J., Wilkins-Haug, L., \& Lieberman, E. (2004). Expectant Management in Spontaneous Preterm Premature Rupture of Membranes between 14 and 24 Weeks' Gestation. Journal of Perinatology, 24 (10), 611-616. doi: 10.1038/sj.jp.7211163.

[7] Kibel, M., Asztalos, E., Barrett, J., Dunn, M., Tward, C., Pittini, A. and Melamed, N., 2016. Outcomes of Pregnancies Complicated by Preterm Premature Rupture of Membranes Between 20 and 24 Weeks of Gestation. Obstetrics \& Gynecology, 128 (2), pp. 313-320.

[8] Goldenberg, R., Hauth, J. and Andrews, W., 2000. Intrauterine Infection and Preterm Delivery. New England Journal of Medicine, 342 (20), pp. 1500-1507.

[9] Bloom, S. (2001). Recurrence of preterm birth in singleton and twin pregnancies. Obstetrics \& Gynecology, 98 (3), 379385. doi: 10.1016/s0029-7844(01)01466-1.

[10] Lewis, D., Adair, C., Weeks, J., Barrilleaux, P., Edwards, M. and Garite, T., 1999. A randomized clinical trial of daily nonstress testing versus biophysical profile in the management of preterm premature rupture of membranes. American Journal of Obstetrics and Gynecology, 181 (6), pp. 1495-1499.

[11] Asadi N, Faraji A, Keshavarzi A, Akbarzadeh-Jahromi M, Yoosefi S. Predictive value of procalcitonin, C-reactive protein, and white blood cells for chorioamnionitis among women with preterm premature rupture of membranes. International Journal of Gynecology \& Obstetrics. 2019; 147 (1): 83-88.

[12] Zaidi H, Lamalmi N, Lahlou L, Slaoui M, Barkat A, Alamrani $\mathrm{S}$ et al. Clinical predictive factors of histological chorioamnionitis: case-control study. Heliyon. 2020; 6 (12): e05698.

[13] Redline R, Faye-Petersen O, Heller D, Qureshi F, Savell V, Vogler C. Amniotic Infection Syndrome: Nosology and Reproducibility of Placental Reaction Patterns. Pediatric and Developmental Pathology. 2003; 6 (5): 435-448.

[14] Mura T, Picaud J, Larroque B, Galtier F, Marret S, Roze J et al Cognitive Impairment at Age 5 Years in Very Preterm Infants Born Following Premature Rupture of Membranes. The Journal of Pediatrics. 2013; 163 (2): 435-440. e2.

[15] Manuck T, Maclean C, Silver R, Varner M. Preterm premature rupture of membranes: does the duration of latency influence perinatal outcomes?. American Journal of Obstetrics and Gynecology. 2009; 201 (4): 414. e1-414. e6.

[16] Melamed N, Hadar E, Ben-Haroush A, Kaplan B, Yogev Y. Factors affecting the duration of the latency period in preterm premature rupture of membranes. The Journal of MaternalFetal \& Neonatal Medicine. 2009; 22 (11): 1051-1056. 